\title{
Perimetric visual field and functional MRI correlation: implications for image-guided surgery in occipital brain tumours
}

\author{
F-E Roux, D Ibarrola, J-A Lotterie, F Chollet, I Berry
}

\begin{abstract}
Objective-To compare the results of visual functional MRI with those of perimetric evaluation in patients with visual field defects and retrochiasmastic tumours and in normal subjects without visual field defect. The potential clinical usefulness of visual functional MRI data during resective surgery was evaluated in patients with occipital lobe tumours.

Methods-Eleven patients with various tumours and visual field defects and 12 normal subjects were studied by fMRI using bimonocular or monocular repetitive photic stimulation $(8 \mathrm{~Hz})$. The data obtained were analyzed with the statistical parametric maps software $\left(p<10^{-8}\right)$ and were compared with the results of Goldmann visual field perimetric evaluation. In patients with occipital brain tumours undergoing surgery, the functional data were registered in a frameless stereotactic device and the images fused into anatomical three standard planes and three dimensional reconstructions of the brain surface.
\end{abstract}

Results-Two studies of patients were discarded, one because of head motion and the other because of badly followed instructions. On the remaining patients the functional activations found in the visual cortex were consistent with the results of perimetric evaluation in all but one of the patients and all the normal subjects although the results of fMRI were highly dependent on the choices of the analysis thresholds. Visual functional MRI image guided data were used in five patients with occipital brain tumours. No added postoperative functional field defect was detected.

Conclusions-There was a good correspondence between fMRI data and the results of perimetric evaluation although dependent on the analysis thresholds. Visual fMRI data registered into a frameless stereotactic device may be useful in surgical planning and tumour removal. (F Neurol Neurosurg Psychiatry 2001;71:505-514)

Keywords: magnetic resonance imaging; perimetric evaluation; visual cortex; neuronavigation; occipital tumour

Dr F-E Roux

rouxfran@compuserve.com

Received 9 November 2000 and in revised form

4 March 2001

Accepted 10 May 2001 It has been shown recently that functional MRI (fMRI) with blood level oxygen dependent (BOLD) contrast was capable of depicting functional areas in patients with brain tumours, providing functional information complementary to the morphological studies. ${ }^{1-4}$ Thus, fMRI has been used in patients with brain tumours, for surgical planning, ${ }^{1-4}$ surgery for epilepsy, ${ }^{4}$ or to detect functional reorganisations induced by functional damage. ${ }^{5}$ It has also been shown that registration of fMRI data in a frameless stereotactic device (functional image guided surgery) for removal of cerebral tumours in eloquent sensorimotor areas could enhance the patients' chances of successful treatment. $^{2}$ Guided by these functional data, cortical brain mapping in these regions is easier, facilitating sparing of functional areas. ${ }^{2}$ The use of cortical brain mapping is, however, restricted to patients who undergo open brain surgery in sensorimotor or language regions. Until recently, in occipital lobe tumours or tumours affecting the afferent visual system tumour, data concerning the visual areas were in clinical practice restricted to visual field assessment. Kollias et al showed that fMRI could be used to detect functional visual areas in patients with brain tumours and visual field defects. ${ }^{6}$ The ability to see the effects of occipital tumours on the visual cortex could be useful to the neurosurgeon in surgical planning. Furthermore, registered in a frameless stereotactic device these fMRI visual data could be used to localise intraoperatively functional visual areas.

The goal of this study was firstly to confirm that the visual field defects identified by perimetric examination were consistent with visual fMRI data in order to use visual fMRI in clinical practice. We thus analyzed and compared the visual fMRI data of volunteers (without visual field defect) and patients (with visual field defects) with brain tumours in the occipital lobes or in the afferent visual system. Secondarily, in patients requiring occipital lobe tumour removal, visual fMRI data were used registered in a neuronavigational system.

\section{Material and methods}

PATIENTS

The group consisted of 11 patients with brain tumours and visual field defects, operated on at our institution between June 1999 and July 2000. Eight men and three women were prospectively studied, ranging in age from 42 to 75 years (mean 56). A visual field defect caused by a cerebral occupying lesion was the selection criterion for participation in this study. Clinical evaluation in each patient included complete neuro-ophthalmological examination, Gold- 
Table 1 Patient characteristics, histological diagnoses, visual field defects, fMRI results and type of operation performed

\begin{tabular}{|c|c|c|c|c|c|}
\hline Patients & Age, sex & Tumour type, location & Visual field defect & FMRI result ( $p<10^{-8}:$ SPM analysis $)$ & Operation \\
\hline 1 & $48, M$ & Astrocytoma, grade III, right lingual gyrus & Left HLH & No right visual cortex activation & $\begin{array}{l}\text { Tumour } \\
\text { removal }\end{array}$ \\
\hline 2 & $52, \mathrm{M}$ & Astrocytoma, grade III, left lingual gyrus & Right HLH & No left visual cortex activation ${ }^{\star}$ & $\begin{array}{l}\text { Tumour } \\
\text { removal }\end{array}$ \\
\hline 3 & $64, M$ & Meningioma, left and right cuneus & $\begin{array}{l}\text { Right inf lat hom } \\
\text { quadranopsy }\end{array}$ & No left sup visual cortex activation & $\begin{array}{l}\text { Tumour } \\
\text { removal }\end{array}$ \\
\hline 4 & $75, M$ & Glioblastoma, left occipital lobe & Right HLH & NP & Biopsy \\
\hline 5 & $42, \mathrm{~F}$ & Astrocytoma, grade III, right trigone & Left HLH & No right visual cortex activation & Biopsy \\
\hline 6 & $54, \mathrm{M}$ & Meningioma, left posterior lingual gyrus & $\begin{array}{l}\text { Right sup lat hom } \\
\text { quadranopsy }\end{array}$ & No left inf visual cortex activation & $\begin{array}{l}\text { Tumour } \\
\text { removal }\end{array}$ \\
\hline 7 & $69, M$ & Glioblastoma, right occipital lobe & Left HLH & $\begin{array}{l}\text { Right and left asymmetric visual cortex } \\
\text { activation }\end{array}$ & $\begin{array}{l}\text { Tumour } \\
\text { removal }\end{array}$ \\
\hline 8 & $58, M$ & $\begin{array}{l}\text { Astrocytoma, grade III, right trigone and } \\
\text { parietal lobe }\end{array}$ & $\begin{array}{l}\text { Left inf lat hom } \\
\text { quadranopsy }\end{array}$ & No right sup visual cortex activation & Biopsy \\
\hline 9 & $51, \mathrm{~F}$ & Intra and suprasellar hypophysal adenoma & Bitemporal hemianopsy & $\begin{array}{l}\text { Left eye stimulation : no right visual cortex } \\
\text { activation }{ }^{\star} \\
\text { Right eye stimulation : no left visual cortex } \\
\text { activation }{ }^{\star}\end{array}$ & $\begin{array}{l}\text { Tumour } \\
\text { removal }\end{array}$ \\
\hline 10 & $53, \mathrm{~F}$ & Astrocytoma, grade III, left temporal lobe & $\begin{array}{l}\text { Right sup lat hom } \\
\text { quadranopsy }\end{array}$ & No right inf visual cortex activation $\dagger$ & $\begin{array}{l}\text { Tumour } \\
\text { removal }\end{array}$ \\
\hline 11 & $50, M$ & Glioblastoma, right trigone & Right HLH & Head motion: discarded & Biopsy \\
\hline
\end{tabular}

$\mathrm{HLH}=$ Homonymous lateral hemianopsy; sup lat hom=superior lateral homonymous; inf lat hom=inferior lateral homonimous; SPM=statistical parametric maps; $\mathrm{NP}=$ not performed

*Significant analysis threshold: $\mathrm{p}<10^{-9}$.

†Significant analysis threshold: $\mathrm{p}<0.001$

within the week of fMRI. In each case, neuroophtalmological examination documented various visual field defects: quadranopsia and homonymous or bitemporal hemianopsia. The specific histopathological nature of each of these space occupying lesions was established by open surgery (seven patients) or by biopsy (four patients). The patient characteristics, histological diagnoses, visual field defects, and type of operation performed are summarised in table 1 .

\section{VOLUNTEERS}

Twelve normal volunteers (range 22 to 55 years; mean 27) were also studied with fMRI as a control group. Each normal volunteer also had a complete neuro-ophthalmological examination and Goldmann kinetic perimetry that confirmed that each volunteer had a visual acuity at least of $7 / 10$ or better and no history of ophthalmological problems. No subject was used more than once. The controls were not age matched. Permission to carry out this study was obtained from the subject in accordance with guidelines approved by the regional ethics committee. All 12 controls were studied with eyes open and closed following the instructions of the acquisition procedure and were given the same instructions as patients.

\section{STIMULUS CONDITION AND FMRI DATA}

ACQUISITIONS

We achieved visual activation using a computer operated stroboscope generating white-red light flashing at a frequency of $8 \mathrm{~Hz}$. Flash and intensity were constant throughout the stimulation cycles and the whole study (patients and volunteers). Visual stimuli were given by special MRI compatible glasses (Resonance Technology inc, Northridge, CA, USA). During the stimulation periods, the subjects were instructed to open their eyes and to stare at the centre of the light emitting source while during the resting state the subjects were kept in the dark with their eyes closed. Patients were positioned in the head coil of a $1.5 \mathrm{~T}$ Magnetom Vision $^{\circledR}$ MR scanner (Siemens ${ }^{\circledR}$, Erlangen,
Germany). Optimisation of the magnetic field was performed with the automatic map shim procedure to reach a gradient tolerance of $0.001 \mathrm{mT} / \mathrm{m}$. fMRI data were obtained using an GE-EPI single shot sequence ( $\mathrm{TE}=60 \mathrm{~ms}$, $\mathrm{FA}=90^{\circ}$, slice number $=10$, matrix size $=64 \times 64$, $\mathrm{FOV}=200 \mathrm{~mm}$, slice thickness $=5 \mathrm{~mm}$, distant factor $=0.5 \mathrm{~mm}$ ). The 10 slices were positioned parallel to the anterior commissure and the posterior commissure (AC/PC) axis on the occipital lobes. We used an automated shim procedure to improve the magnetic field homogeneity. We used a head holder with headphones and special padding that minimised head motion. A staff member was always present near the patient during the acquisitions to control the procedure and to encourage the patient to do the task as best he could. Usually, the whole fMRI procedures lasted 10 minutes. During the procedure, the patient alternated periods of rest (eyes closed) and periods of activation (eyes open). Each period lasted 30 seconds while 10 images were acquired every 3 seconds. Alternative rest and activation periods were repeated four times with each procedure beginning by a period of rest. Each period was controlled vocally by the headphones.

Postoperatively, two patients (patients 3 and 9) had a control visual fMRI study with the same protocol conditions.

\section{fMRI ANALYSIS}

The fMRI data were analyzed with the statistical parametric map (SPM) 96 software $^{7}$ and performed on a Sun SPARC workstation (Mountain view, CA, USA). The first three images of each run were discarded to allow signal stabilisation and the remaining 77 volumes of 10 slices were realigned to correct the subject movement during scanning using the first volume of images as reference. Then the detection of the activated voxels was performed on a pixel by pixel basis. Supposed changes in neuronal activity were detected using the blood oxygen level dependent (BOLD) technique. If large movements of the head occurred $(>5$ 
$\mathrm{mm}$ ), motion artifacts were not corrected and the data were excluded from analysis. We used the general linear model implemented in SPM 96 where conditions (rest or activation) stand for independent variables after global normalisation to cancel differences among scans. Conjunction analysis of normal subjects was done according to SPM96 conditions. Voxels were considered initially as significant if $\mathrm{p}<10^{-8}$.

FUNCTIONAL GUIDED SURGERY PROCEDURE

For patients shortlisted for surgery, $1 \mathrm{~mm}$ anatomical slices were acquired to be used in the frameless stereotactic neuronavigational device. Local distortions of the surface of the gyri produced by the tumour were seen on the three standard anatomical planes (coronal, axial, and sagittal) and on three dimensional reconstructions. This allowed preoperative and intraoperative computer assisted assessment of the relations between the tumour and the important landmarks of gyral anatomy. The surfaces of the brain were reconstructed from a three dimensional data set obtained with a 3DMPRAGE sequence $(\mathrm{TR}=15 \mathrm{~ms}, \mathrm{TE}=7 \mathrm{~ms}$, $\mathrm{FA}=12^{\circ}, 128$ partitions, $\mathrm{FOV}=300 \mathrm{~mm}$, matrix size $=256 \times 256$, slab thickness $=150 \mathrm{~mm}$, voxel size $\left.=1 \times 1 \times 1 \mathrm{~mm}^{3}, \mathrm{NA}=1, \mathrm{TA}=14 \mathrm{~min}\right)$. The centre of the three dimensional block was positioned according to the axis joining the AC/PC. These three dimensional sequences lasted 14 minutes each.

For the patients with occipital lobe tumours, the fMRI images were reformatted to be integrated into a frameless stereotactic device (Stealth Station, Sofamor Danek, Surgical navigation technologies, Broomfield, $\mathrm{CO}$, USA) using the ANALYZE software (Mayo Clinic, Rochester, MN, USA), the ImMerge software, and the graphic tools of the neuronavigational sytem. The patients were operated on within 1 to 7 days after the preoperative functional brain mapping analysis had been completed. This image processing took about 1 hour to perform (three dimensional reconstruction of the brain, tumour, and functional MRI registrations). The exact locations of functional sites were analysed with the three dimensional reconstructions of the brain surface. In the operating room, patients received a general anaesthetic, and three point head fixation was applied. The image and surgical space registered, the accuracy of the registration confirmed, and the brain exposed in a standard fashion. No cortical and subcortical mapping was used. Our policy was to spare the cortical functional areas found by fMRI during tumour removal. Intraoperative photographs of the brain were taken.

\section{Results}

ANATOMICAL MRI: PATIENTS' RESULTS

Two extra-axial tumours were found (occipital meningiomas). In patient 3 , clear compression of the superior part of the left calcarine fissure was demonstrated by MRI. In comparison with the contralateral side, the left calcarine fissure was probably lowered. Patient 6 had a small meningioma located medially and compressing the left lingual gyrus and the calcarine fissure.
In patient 9, a hypophysial adenoma had bled inducing an acute increase in tumour volume and a bitemporal hemianopsy (old haemorrhage was therefore found at operation during adenoma removal). Four patients had intraxial tumours located far from of the calcarine cortex but with MR evidence for involvement of the optic radiations located in the trigone area (three patients) and medially in the temporal lobe (one patient). The last four patients had pure malignant tumours of the intra-axial occipital lobe with presence of oedema or tumour infiltration making the calcarine fissure sometimes difficult to identify accurately.

\section{fMRI: VOLUNTEERS' RESULTS}

In the group of 12 control subjects, areas of activation were located almost exclusively in the occipital lobes (Brodmann areas 17, 18, and 19). Although centred on the primary visual cortex in the conjunction analysis (fig 1), areas of activation were individually variable in shape and extent (fig 2). Data were analyzed with an analysis threshold of $\mathrm{p}<10^{-8}$ adjusted for all volunteers but one. One visual activation had to be analyzed with a threshold of $p<0.001$ because with a higher analysis threshold no significant visual activation area was detected. The activations were bilateral, along the calcarine fissure. Quantitative analysis between subjects disclosed important total pixel count differences varying from 1 to 200 . The hemispheric difference activation ratio was measured in all subjects. It was between $0 \%$ and $40 \%$ in all volunteers but one. One subject had bilateral primary visual area activation but with a right to left activation ratio of $90 \%$ $\left(\mathrm{p}<10^{-8}\right)$ with no visual field defect on perimetric evaluation. Some activations were also found in the lateral occipital cortex in one subject and in the frontal lobes in another subject.

\section{fMRI: PATIENTS' RESULTS AND PERIMETRIC}

EVALUATION CORRELATION

Among the 11 patients, two studies were discarded, one because of excess motion (patient 11) and the other because the patient could not cooperate during fMRI runs (patient 4). Data were first analysed with an analysis threshold of $\mathrm{p}<10^{-8}$. Areas of activation were found in the mesial portion of the occipital lobes in the presumed anatomical location of the calcarine fissure. As with the normal control subjects, these areas of activation were variable in shape and extent individually. In most cases, the tumour related regional brain deformation prevented us from knowing accurately whether these variations in activation across patients were related to individual anatomy of the calcarine fissure. Few additional regions of activation were detected in the lateral occipital cortex in one patient and in frontal areas in another. Areas of activation on fMRI were sometimes found in infiltrated parenchyma adjacent to tumours. For the analysis threshold chosen, no significant focus of increased activation was found within the tumours. 


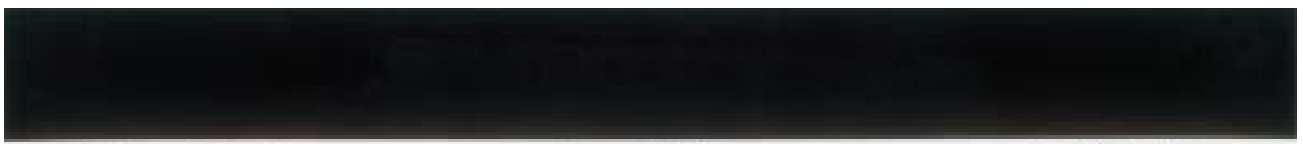

$z=-571111$
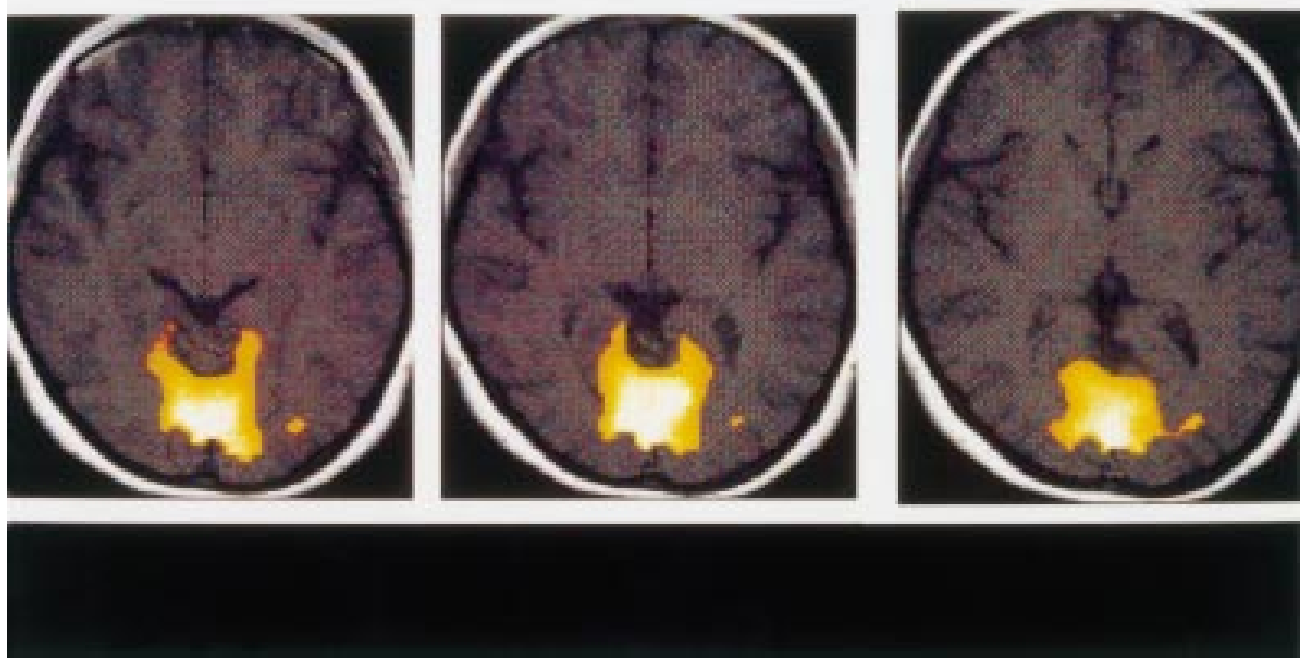

Figure 1 Conjunction analysis of the fMRI visual data of the normal subjects. The primary visual cortex, located along the calcarine fissure is mainly activated. SPM 96 analysis: $p<10^{-8}$.

These significant activations demonstrated within the mesial regions of occipital cortex were analyzed according to the visual field defects of the remaining nine patients. For the initial analysis threshold chosen $\left(\mathrm{p}<10^{-8}\right)$, initial qualitative analysis of both fMRI activations and perimetric evaluation showed consistent results in five patients. In two patients who had left
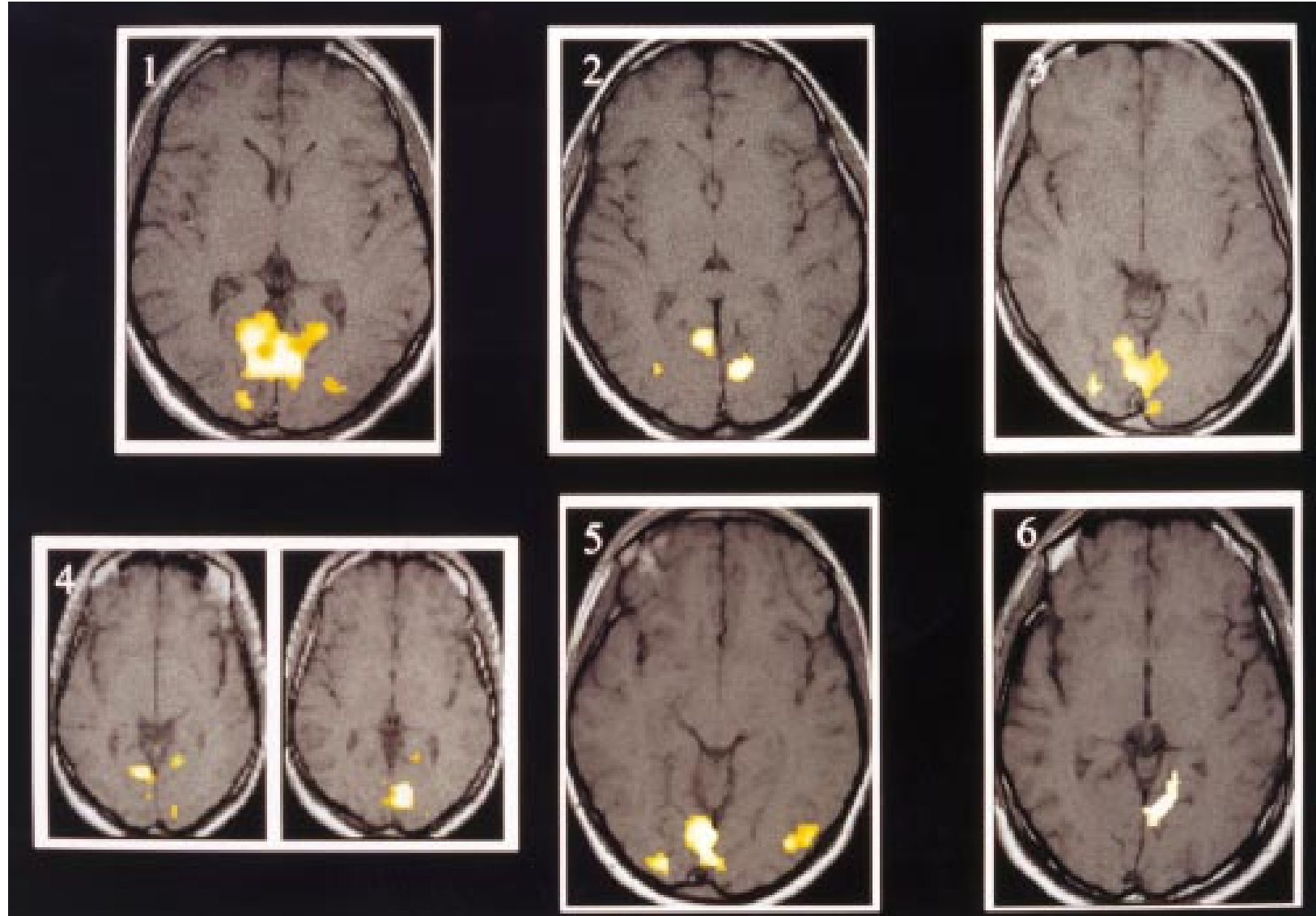

Figure 2 Illustration of the different individual patterns of activation in the normal subjects (no visual field defect). The activated areas of six different subjects are represented. The same activation protocol and the same analysis threshold have been used $\left(p<10^{-8}\right)$. Individual variations of the activated areas are clearly visible. The activations are bilateral, along the calcarine fissure, variable in shape and extent. The total pixel count differences (between subjects) varied from 1 to 200. For 11 subjects, the within subject pixel count disclosed a hemispheric difference (left versus right) activation ratio between $0 \%$ and 40\%. One volunteer (6) had a right to left activation pixel ratio of 1 to 9 without any visual field defect. 


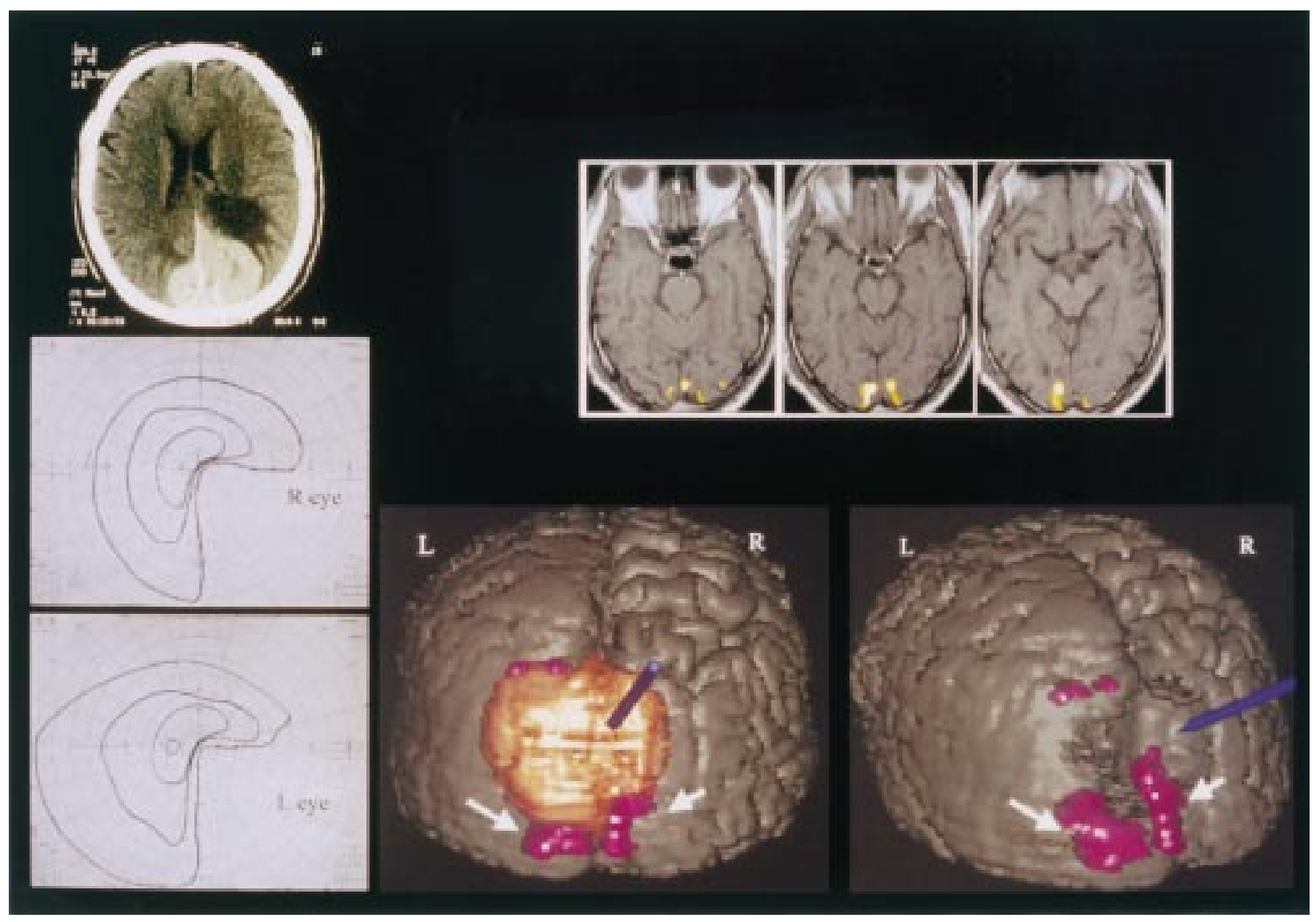

Figure 3 Patient 3: findings obatined in a 64 year old man with occipital meningioma. Goldmann perimetry showing a right inferior congruous quadranopsy. Preoperative CT shows a huge occipital meningioma. Preoperative fMRI findings (binocular stimulation: upper right) on axial planes and registered in a three dimensional reconstruction of the patient's brain surface (lower right) showed that activation is present bilaterally (purple arrows) but is lacking in the left superior part of the visual cortical areas on the three dimensional model.

hemianopsy (patients 1 and 5), no fMRI activation was located contralaterally to the visual field defect. In patients 3 and 8 with respectively right and left inferior quadranopsies, the fMRI activation could be detected bilaterally but no clear activation could be found in the superior contralateral extrastriate V2 or V3 cortices (fig 3). In patient 6 with right superior quadranopsy, no clear activation could be found in the inferior contralateral visual areas. In three other patients (patients 2, 9, and 10), fMRI results were also consistent with those of perimetric examination but with different analysis thresholds. In patients with right lateral homonymous superior quadranopsy due to deafferentation (patient 10), no activation $\mathrm{fMRI}$ data could be found in the inferior left visual cortical areas but the analysis threshold that had to be chosen was less significant than the previous ones $(p<0.001)$. In patient 2 presenting with a right lateral homonymous hemianopsy, a more significant threshold $\left(\mathrm{p}<10^{-9}\right)$ than the initial one had to be chosen to obtain a good correlation between perimetry and fMRI data. We also tested the patient with bitemporal hemianopsy by occluding, successively in two separate fMRI examinations, left and right eyes. We hypothesised that contralateral activation to the activated eye should be absent. As we did for patient 2, we chose a more significant analysis threshold $\left(\mathrm{p}<10^{-9}\right)$. Indeed, no contralateral activation was found when the right and left eye were separately stimulated, also demonstrating the good correlation between fMRI and perimetric evaluation of the visual field, in this case of bitemporal hemianopsy. Finally, patient 7, whose findings on fMRI and those of perimetric evaluation did not correlate, had a clear congruous right homonymous hemianopsy (without macula sparing) but a bilateral activation even with high analysis threshold. Nevertheless, this bilateral activation was asymmetric with a clear right to left ratio of 0.6.

Areas of activation were highly dependent on the analysis threshold chosen. The choice of a less significant analysis threshold ( $p<0.001$, for example) in most patients resulted in an extension of the previous activated areas and appearance of new activated areas. This variation made the qualitative correlation between perimetric visual field evaluation and fMRI data invalid. Three patients had to be analysed with different analysis thresholds than the initial threshold chosen; one (patient 10) with a less significant threshold $(\mathrm{p}<0.001)$ and two (patients 2 and 9) with a more significant one $\left(\mathrm{p}<10^{-8}\right)$ illustrating the threshold importance in the data analysis and its individual variations.

POSTOPERATIVE VISUAL FUNCTIONAL MRI

Patient 3 and patient 9 had a visual fMRI study postoperatively. Patient 3 with persistant postoperative left superior quadranopsic homonymous defect had no significant modification of 


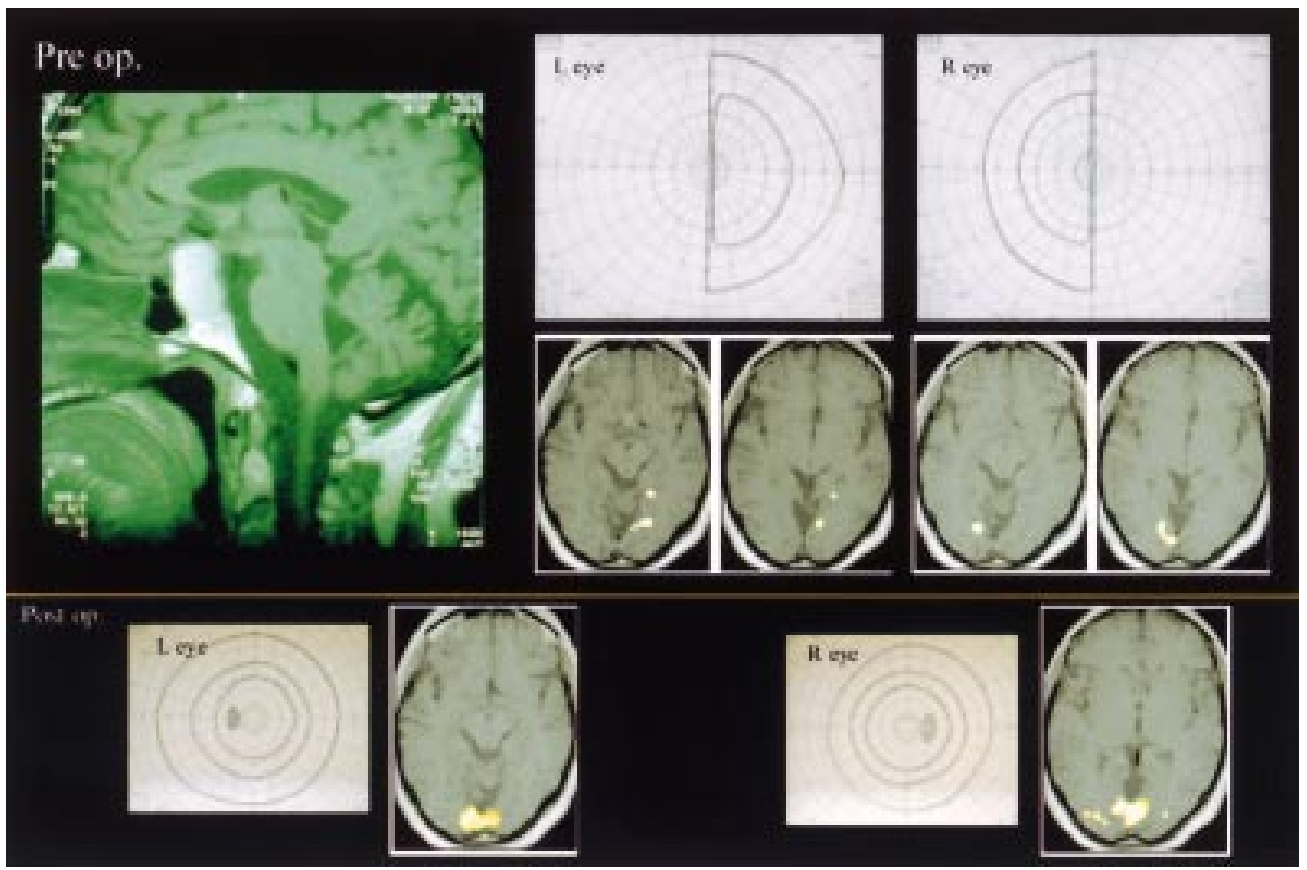

Figure 4 Patient 9: findings obtained in a 51 year old woman with intrasellar and suprasellar hypophysal adenoma and bitemporal hemianopsy. Preoperative findings: sagittal T1 weighted MR image demonstrating the suprasellar extension of the adenoma. Goldmann perimetry shows a typical bitemporal hemianopsy. fMRI findings (monocular stimulation): the left eye stimulation gave no activation of the right visual cortex, the right eye stimulation gave no left visual cortex activation. The patient was operated on rapidly and the tumour removed (transsphenoidal approach). Postoperative findings (1 month): Goldmann perimetry showing an almost complete recovery of the visual field defect. fMRI findings (monocular stimulation): the left eye stimulation and right eye stimulation gave bilateral stimulation of the visual cortex.

the activation pattern of his visual areas in comparison with the preoperative fMRI study. Patient 9, who fully recovered from her bitemporal hemianopsy, had postoperatively bilateral visual area activation during left and right monophotic activation (fig 4 ).

FUNCTIONAL CLINICAL RESULTS

Among the patients with visual field defects tested, six had occipital lobe tumours. Five of these patients underwent gross tumour removal using visual functional data integrated in a frameless stereotactic device. One had a stereotactic biopsy of his tumour (patient 4). Five other patients with brain tumours of various locations had visual field defects by deafferentation. Patient 9 with an intrasellar and suprasellar adenoma was operated on using a rhinoseptal approach. Patient 10 underwent removal of her temporal tumour followed by radiotherapy. Three other patients with tumours in various locations had only a stereotactic biopsy and radiotherapy, and eventually chemotherapy. In our five patients operated on using functional and anatomical data integrated in a neuronavigational device, no postoperative added visual field defect was found. Visual field improved in one patient 2 months postoperatively, probably because of the decompressive effect of tumour removal. There were no relevant complications related to surgery.

\section{Discussion}

CORRELATIONS OF FMRI DATA WITH PERIMETRIC EXAMINATION

Since the development in the early 1990s of the fMRI brain mapping technique, the need to correlate fMRI with established electrophysiological tools has given rise to several studies, especially in motor areas. ${ }^{3}$ Few anatomical MRI studies have examined the relations between cerebral lesions and striate cortex..$^{8-10}$ In primary visual areas, preliminary fMRI data obtained in a few patients with optic nerves, chiasmatic or retrochiasmatic lesions, and visual field defects have displayed a correlation between the fMRI data and those of the perimetric evaluations. ${ }^{11}$ The ability of fMRI to detect visual field defects that are caused not only by tumours of the primary visual cortex, but also by lesions producing visual field defects by deafferentation (whereas the occipital cortex is intact) has also been shown by Kollias $e t a l^{6}$ in all but one of their 10 patients, using Goldmann perimetric evaluation. The advantages of correlation with perimetric visual field evaluation are numerous; as it is easy to perform, not expensive and non-invasive, it can also be used for control groups. We also found a good agreement between fMRI data and perimetric evaluation, although not $100 \%$. The reasons for this discrepancy are probably multifactorial. The reliability of the method of reference (perimetric evaluation) can first be questioned. The reliability of subjects to perform perimetry correctly necessitates an adequate visual field examination. ${ }^{12}$ False positives and false negatives in the visual field determination ${ }^{12}$ could lead to misinterpretation of functional correlations. The sensitivity of MRI, especially in these pathological conditions, ${ }^{13}$ including distorted anatomy, and technical issues are also potential sources of error in the correlations. High between subject variability for the same activation task, not only in 
patients (that can be explained by their different pathological states) but also in the control group can be also questioned. ${ }^{14-16}$ Some authors found, using visual fMRI, individual variations of the visual areas among subjects and with time over successive periods attributed, among other factors, to attention, eyelid position, and physiological fatigue. ${ }^{14}$ Among our normal subjects quantitative analysis of the activated pixels also showed important differences for the same analysis threshold and for the same activation task.

The exact representation of the visual field in the occipital lobes has been a matter of debate. Positron emission tomography studies,${ }^{17}{ }^{18}$ anatomical correlation ${ }^{10}$ between anatomical MRI (or CT), and now the increasing use of fMRI in brain mapping ${ }^{11}$ have contributed to increasing our knowledge on the functional organisation of the occipital lobes. Primary visual areas are centred along the calcarine fissure. As already mentioned by other authors ${ }^{9}{ }^{14}$ there is considerable variation in the surface area and extent of the striate cortex ${ }^{8}$ and our findings in both normal subjects and patients confirm this statement. Functionally, there is an increase in eccentricity from posterior visual areas to more anteriorly located medial areas. It is established that the central $10^{\circ}$ of visual fields are represented by at least $50 \%-60 \%$ of the posterior striate cortex. ${ }^{8920}$ Although the macular representation is still a matter of debate, Gray et al concluded that the foveal region was unilaterally represented in the primary human visual cortex $x^{20}$ on the basis of correlating the findings of MRI and visual field defects in patients with occipital lobe injury. It is also established that the horizontal meridian is represented deep within the calcarine banks at the fissure base. ${ }^{20} 21 \mathrm{~A}$ lesion in the upper bank of the calcarine cortex causes a partial deficit in the contralateral inferior quadrant of the visual field. Patient 3 had this pattern of lesion with a meningioma leaving on both sides of the sagittal sinus but with important compression toward the superior bank of the left calcarine fissure. He had a right inferior homonymous quadranopsic defect probably due to damage of the extrastriate cortex or damage to the primary visual cortex over the representation of the horizontal meridian at the base of the calcarine fissure. During the operation, we therefore found an irregular dissection plane and a deeply modified cerebral parenchyma. Postoperative visual fMRI, done 2 months after operation when the visual field defect had not improved, confirmed the preoperative functional finding. On the contrary, a lesion of the inferior bank of the calcarine fissure causes a partial deficit in the superior quadrant of the contralateral visual field, as in patient 6 . The contralateral hemifield of vision is impaired with more important lesions of the visual cortex (that include both superior and inferior part of the calcarine fissure) as in our patients $1,2,4$, and 7 . Patient 9 is very interesting. She had bitemporal hemianopsia caused by a suprasellar extension of a hypophysal adenoma. We used left and right monocular photic stimulation (in two different
fMRI runs) because simultaneous stimulation of both eyes would have led to bilateral activation of the visual cortex and no correlation with perimetric evaluation would have been possible. ${ }^{11}$ Left photic stimulation led only to activation of the left visual cortex whereas right photic stimulation led only to activation of the right visual cortex. The nasal retina fibres, impaired by the suprasellar extension of the tumour at the chiasmatic decussation, had prevented any contralateral activation. Postoperative fMRI, done when the patient had recovered from her bitemporal hemianopsia, showed (with exactly the same examination conditions) bilateral activation during left and right monophotic activation.

We also found that fMRI data were correlated with perimetric evaluation in lesions producing visual field defects by deafferentation. In relation to this result, it has therefore been shown with PET that ischaemic damage limited to the optic radiations induced decreased metabolism in the striate cortex corresponding to the visual field defect. ${ }^{17}$ Anatomically, after leaving the lateral geniculate nucleus the optic radiation fibres curve into the temporal lobe (Meyer's loop). Global lesions of the optic radiation produce contralateral homonymous hemianopsy as in patients 5 and 11 . But whether an optic radiation lesion could produce quadranopsic defects has been debated ${ }^{9}$ leading to the issue as to whether visual radiation fibres are so strictly organised as to produce a quadranopsic defect. We had two patients with quadranopsy by deafferentation (patients 8 and 10). Because the resolution of MRI in studying the calcarine fissure is lower in the axial than in the coronal planes, we integrated the functional data in a three dimensional individual reconstruction of the brain to ensure that the visual field defects seen with fMRI were correlated with the perimetric evaluation. We found that a lesion of these fibres during temporal lobe surgery, as in our patient 10, caused an upper contralateral quadrantic anopsia (visual defect by deafferentation). But in this case, there was no strict respect of the vertical and horizontal meridians. The second patient with a quadrantic defect was patient 8 with a right parietal tumour. There was in this case a congruous left inferior homonymous quadranopsy with respect to the horizontal meridian (fig 5). In these two patients with quadranopsies, the fMRI activations could be detected bilaterally but no clear activation could be found in the superior (patient 8) or inferior (patient 10) contralateral extrastriate V2 or V3 cortices.

\section{METHODOLOGICAL ISSUES}

It is fundamental to understand the methodological issues related to the use of fMRI, especially in these non-physiological conditions. Technical problems such as echoplanar distortion, paradigm choices, analysis thresholds, movement artifacts due to slight head movements, brain pulsatility, and "vein effects" have been evoked in many fMRI studies. ${ }^{1622-26}$ The changes with the rate, ${ }^{18}$ with the patient's ability to do the task, and the specialisation of the 


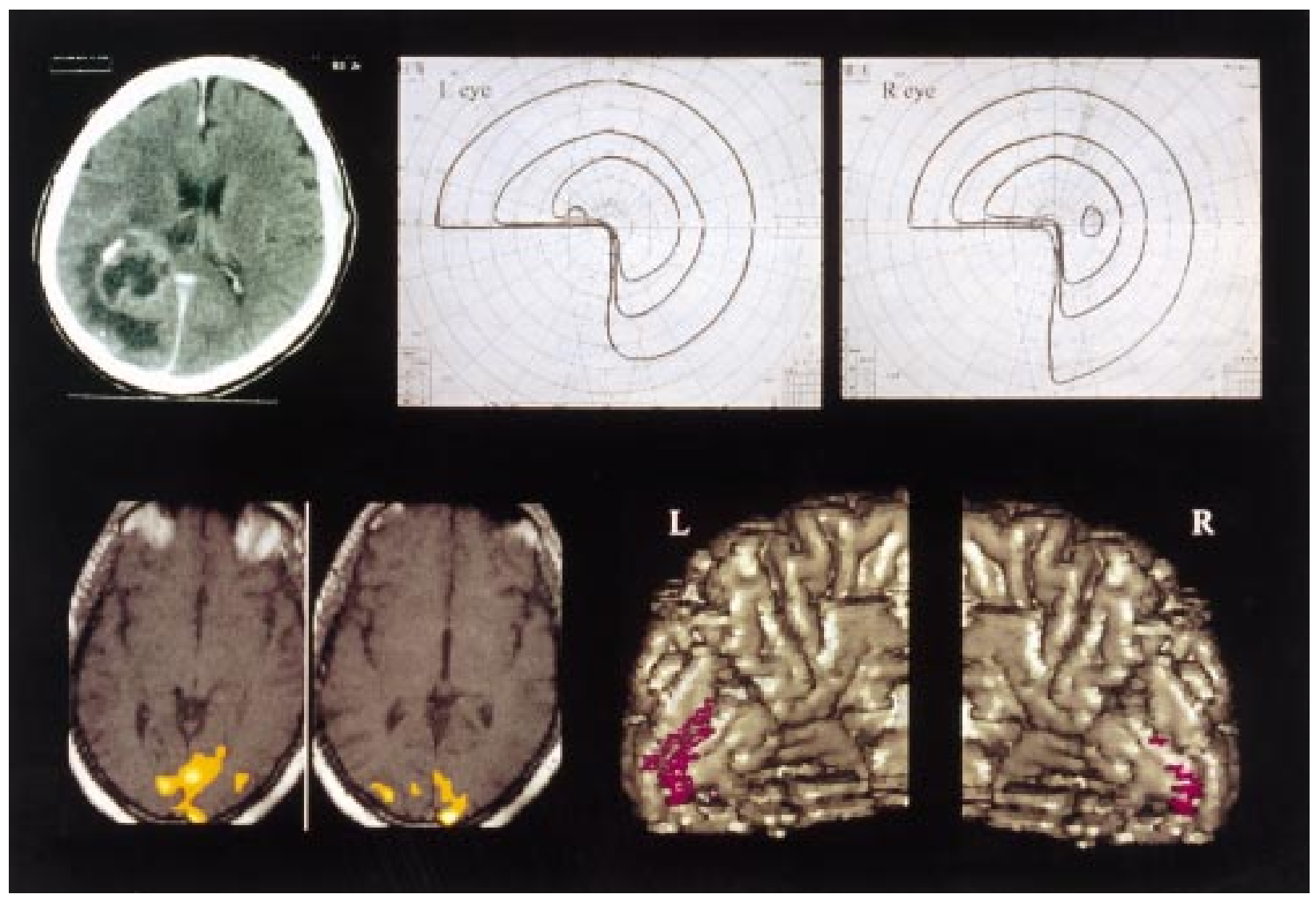

Figure 5 Patient 8: findings from a 58 year old man with right parietal grade III astrocytoma and left inferior congruous quadranopsy (upper right). Preoperative CT showing the parietal lobe tumour (upper left). Preoperative fMRI findings (binocular stimulation) on axial planes and registered in a three dimensional reconstruction of the patient's brain surface: activation is present bilaterally (purple) but is lacking in the right superior part of the visual cortical areas through the supracalcarine V2 and V3 areas.

cerebral structure studied ${ }^{27}$ can also make the analysis of fMRI data extremely difficult. Fox et al concluded that the stimulus rate was a significant determinant of regional cerebral blood flow response in the visual cortex, ${ }^{18}$ the response peaking at about $8 \mathrm{~Hz}$ and then declining. These findings were important in the choice of the rate of our visual paradigm (8 $\mathrm{Hz}$ ). The capability of this paradigm to easily detect primary visual areas and especially its simplicity of utilisation in patients were also important in the paradigm choice. Possibly more elaborate activation paradigms would also have detected not only primary but also secondary visual areas. But the use of such paradigms is less easy in clinical practice and the real clinical benefit of the utilisation of more complex paradigms is unknown.

Intraoperative cortical mapping is one of the methods that can validate fMRI data in patients with brain tumours. ${ }^{3}$ It is in these particular conditions, prone to controversies, that the validation of fMRI data should be the most useful. The significant thresholds chosen to generate activation maps of a function are arbitrary and we have shown that they can be, in some part, individual. It is precisely that discrepancy between the statistical map and the "true" map of a function that requires validation. The individual variations of the analysis threshold are potentially disturbing not only in research but also for clinical application of the fMRI technique. Such individual variation can be of importance for clinical applications such as surgical planning. The individual correlation with the Goldmann perimetric visual field is one of the methods that can easily validate visual fMRI data. Less easily done, the comparison of the visual fMRI data by visual PET could be one of the means of validating the fMRI data. Morever, the intrinsic differences between both techniques in terms of neurophysiology could not solve the problem. Unfortunately, intraoperative cortical mapping cannot be used routinely for visual areas and the intraoperative validation of the statistical maps given by the analysis of visual fMRI remains difficult. ${ }^{28}{ }^{29}$ It is possible to obtain open brain mapping with visual evoked potentials and light emitting diode goggles and cortical grid recordings. ${ }^{28}$ But this brain mapping method is time consuming, rather expensive, and cannot be routinely performed in most neurosurgical departments. The deep intrasulcal location of the visual activation could also present a major problem for cortical grid brain mapping. Occipital and adjacent cortices mapping with electrical cortical stimulation has also been carried out using subdural grid electrodes in 23 patients with epilepsy by Lee et al. ${ }^{29}$ This method necessitates two operations (placement of the grid and removal), and is time consuming; although perhaps suitable for epileptic patients, its use in patients with brain tumours seems difficult. To date, the clinical use of visual fMRI data in neurosurgery must be done 
cautiously and with these interpretative issues in mind.

FUNCTIONAL IMAGE GUIDED SURGERY

In the past few years, the development of image guided neurosurgery has maximised resection in patients with brain tumours and has aided in surgery for epilepsy. Although the timing and the indications of surgery in brain tumours can be controversial, sparing functional areas during tumour resection is accepted as one of the principles in brain tumour surgery. ${ }^{30-32}$ Because considerable variation occurs among individual patients in relative position and size of different cortical visual areas $^{33}$ the exact position of these visual areas cannot be predicted only on the basis of anatomical landmarks. The regional anatomy can be distorted by a tumour mass effect leading to mislocalisation of functional areas. Individual evaluation must be the rule in this field. The technique of visual functional data acquisition is short (10 minutes), well tolerated by the patient, and can be performed after conventional anatomical runs. Data processing and registration in a neuronavigational system can usually be done in 1 hour. Nevertheless, if fMRI is able to detect visual field defects caused by tumours remote from the occipital areas (visual field defect by deafferentation), fMRI has little or no sensitivity for subcortical area changes and particularly for white matter tracts. As we saw in some of our patients, the tumourous or surgical interruption of these tracts leads to visual field defect. There is no direct surgical application of fMRI in these cases.

Pure technical problems can be significant in this clinical application of visual fMRI. Our series of occipital tumours is too small and the visual fMRI image guided surgery application too novel to master all technical related issues. But experience with larger series using the same protocols in motor areas can be useful. ${ }^{3}$ To be used for neurosurgical purposes, fMRI echo planar images $\left(\mathrm{T} 2^{\star}\right)$, anatomically imprecise, must be integrated into anatomical slices. The registration of fMRI raw data in the three dimensional neuronavigation bloc is dependent on software algorithms and on the capability of fusing the same functional and anatomical slices that do not have the same pixel resolution. Anatomical distortion, difference in slice thickness, and integration into the anatomical slices of the frameless stereotactic device can be potential sources of mislocalisation. Strict image processing and neuronavigational registration are essential but probably do not solve all these technical issues. Our fMRI acquisitions in axial plane are probably not the best way to study the calcarine sulci, that are better seen on coronal or sagittal images. ${ }^{10}$ However, the registration in the neuronavigational device is far easier to perform using axial planes.

Some authors have found, using cortical brain mapping ${ }^{30}$ or motor fMRI, ${ }^{123}$ that functioning motor, sensory, or language tissue could be located in surrounding infiltrated brain as well as within frankly evident tumour. We also noted such phenomena with the visual functional cortex. Functional visual activation has been seen within T2 hyperintense peritumoural areas, or next to the tumour in the surrounding oedema generally considered as infiltrated by tumourous cells. Such a finding poses a question about the extent of resection: preservation of brain function (as demonstrated by fMRI) should be judged in terms of resection maximisation. How far the resection regarding the functional tissue should be done-and the possibility of compensatory phenomena with time-is still unknown. Furthermore, an absence of detection of a functional tissue by fMRI would not imply that no viable functional tissue could be potentially present (false negative) in the boundary of a tumour. ${ }^{34}$ This is particularly important in patients with no visual functional activation detected (hemianopsic patients for instance) in whom, because of their visual deficit, no functional data are available. This was the case in our patient 2. The possibilities of visual recovery would be impaired by too confident resection of these supposed "non-eloquent" areas. This is one of the major limitations for the use of visual fMRI data in a neuronavigational system.

\section{Conclusions}

Although the results of visual fMRI in most of our patients and normal subjects were consistent with those of visual field perimetric evaluation, they depended on the analysis threshold chosen, which remained individual. Although reliable data exist in the use of motor $\mathrm{fMRI}^{3}$ for patients with rolandic tumours, this is still not the case for visual fMRI. If it seems proved that perimetric visual field defects are correlated with visual fMRI data in most cases, the use of intraoperative visual fMRI data is still hindered by methodological issues. More studies are needed to overcome methodological problems such as choice of the analysis threshold and fMRI data validation for a more routine application of visual fMRI data. Nevertheless, in selected patients, the visual fMRI data registration in a frameless stereotactic device can be potentially useful in assessing intraoperatively individual relations between function and occipital lobe tumours.

We thank Professor Tremoulet and Dr Gigaud for referring patients to us.

1 Atlas SW, Howard II RS, Maldijian J, et al. Functional magnetic resonance imaging of regional brain activity in patients with intracerebral gliomas: findings and implica38 .

2 Schulder M, Maldijian JA, Liu WC: Functional imageguided surgery of intracranial tumours located in or near guided surgery of intracranial tumours located in or n
the sensorimotor cortex. $\mathcal{F}$ Neurosurg 1998;83:412-18.

the sensorimotor cortex. F Neurosurg 1998;83:412-18.
3 Lehéricy S, Duffau H, Cornu P, et al. Correspondance between functional MRI somatotopy and individual brain mapping anatomy of the central brain region: comparison with intraoperative stimulation in patients with brain tumours. F Neurosurg 2000;92:589-98.

4 Stapleton SR, Kiriakopoulos E, Mikulis D, et al. Combined utility of functional MRI, cortical mapping and frameless stereotaxy in the resection of lesions in eloquent areas of brain in children. Pediatr Neurosurg 1997;26:68-82.

5 Cao Y, D'Olhaberriague L, Vikingstad EM, et al. Pilot study of fMRI to assess cerebral activation of motor function after poststroke hemiparesis. Stroke 1998;29:112-22.

6 Kollias SS, Landau K, Khan N, et al. Functional evaluation using magnetic resonance imaging of the visual cortex in patients with retrochiasmatic lesions. $\mathcal{F}$ Neurosurg 1998;89: patients

7 Friston KJ, Holmes AP, Worsley KJ, et al. Statistical parametric maps in functional imaging: a general appoach. Hum Brain Mapp 1995;2:189-210. 
8 McFadzean R, Brosnahan D, Hadley D, et al. Representation of the visual field in the occipital striate cortex. $B r \mathcal{F}$ tation of the visual field in the

9 Horton JC, Hoyt WF. The representation of the visual field in human striate cortex: a revision of the classic Holmes map. Arch Ophthalmol 1991;109:816-24.

10 Kitajima M, Korogi Y, Kido T, et al. MRI in occipital lobe infarcts: classification by involvement of the striate cortex. Neuroradiology 1998, 40:710-15.

11 Miki A, Nakajima T, Takagi M, et al. Detection of visua dysfunction in optic atrophy by functional magnetic resonance imaging during monocular visual stimulation. Am f Ophthalmol 1996;122:404-15.

12 Lee M, Zulauf M, Caprioli J. The influence of patient reliability on visual field outcome. Am $\mathcal{f}$ Ophthalmol 1994;117:756-61.

13 Holodny AI, Schulder M, Liu WC, et al. Decreased BOLD functional MR activation of the motor and sensory cortices adjacent to a glioblastoma multiforme: implications for image-guided neurosurgery. AfNR Am ₹ Neuroradiol 1999; 20:609-12.

14 Liu GT, Fletcher DW, Bishop RJ, et al. Variability in visual cortex activation during prolonged functional magnetic resonance imaging. F Neuroophtalmol 1998;18:258-62.

15 Hedera P, Wu D, Collins S, et al. Sex and electroencephalographic synchronization after photic stimulation predic signal changes in the visual cortex on functional $\mathrm{MR}$ images. Am $\mathcal{F}$ Neuroradiol 1998;19:853-7.

16 McGonigle DJ, Howseman AM, Athwal BS, et al. Variability in fMRI: an examination of intersession differences. Neuroimage 2000;11:708-34.

17 Bosley TM, Rosenquist AC, Kushner M, et al. Ischemic lesions of the occipital cortex and optic radiations: PET. Neurology 1985;35:470-84.

18 Fox PT, Raichle ME: Stimulus rate dependence of regional cerebral blood flow in human striate cortex, demonstrated by PET. 7 Neurophysiol 1984;51:1109-20.

19 McAuley DL, Ross Russel RW. Correlation of CAT scan and visual field defects in vascular lesions of the posterior and visual field defects in vascular lesions of the posterior
visual field pathways. 7 Neurol Neurosurg Psychiatry visual field path

20 Gray LG, Galetta SL, Siegal T, et al. The central visual field in homonymous hemianopia. Evidence for unilateral fovea representation. Arch Neurol 1997;54:312-17.

21 Galetta SL, Grossman RI. The representation of the horizontal meridian in the primary visual cortex. $f$ Neuroophthalmol 2000;20:89-91.
22 Lai S, Hopkins AL, Haacke EM, et al. Identification of vascular structures as a major source of signal contrast in high resolution $2 \mathrm{D}$ and $3 \mathrm{D}$ functional activation imaging of the motor cortex at 1.5T: prelimerary results. Magn Reson Med 1993;33:453-9

23 Maldijian J, Atlas SW, Howard II RS, et al. Functional magnetic resonance imaging of regional brain activity in patients with intracerebral arteriovenous malformations before surgical or endovascular therapy. F Neurosurg 1996; 84:477-83.

24 Menon RS, Ogawa S, Hu X, et al. BOLD based functional MRI at 4 tesla includes a capillary bed contribution: echoplanar imaging correlates with previous optical imaging using intrinsic signals. Magn Reson Med 1995;33:453-9.

25 Hill DLG, Castellano Smith AD, Simmons A, et al. Sources of error in comparing functional magnetic resonance imaging and invasive electrophysiological recordings. 7 Neurosurg 2000;93:214-23.

26 LeRumeur E, Allard M, Poiseau E, et al. Role of the mode of sensory stimulation in presurgical brain mapping in which functional MRI is used. 7 Neurosurg 2000;93:427-31.

27 Sergent J. Brain-imaging studies of cognitive functions. TINS 1994;17:221-7.

28 Schulder M, Holodny A, Liu WC, et al. Functional magnetic resonance image-guided surgery of tumours in or near the primary visual cortex. Stereotact Funct Neurosurg 1999, 73:31-6.

29 Lee HW, Hong SB, Seo DW, et al. Mapping of functional organization in human visual cortex: electrical cortical stimulation. Neurology 2000;54:849-54.

30 Skirboll SS, Ojemann GA, Berger MS, et al. Functional cortex and subcortical white matter located within gliomas. Neurosurgery 1996;38:678-85.

1 Ojemann JG, Miller JW, Silbergeld DL. Preserved function in brain invaded by tumour. Neurosurgery 1996;39:253-9.

32 Haglund MM, Berger MS, Shamseldin M, et al. Cortical localization of temporal lobe language sites in patients with gliomas. Neurosurgery 1994;34:567-76.

33 DeYoe EA, Carman GJ, Bandettini P, et al. Mapping striate and extrastriate visual areas in human cerebral cortex. Proc Natl Acad Sci USA 1996;93:2382-6.

34 Roux FE, Boulanouar K, Ibarrola D, et al. fMRI and cortical brain mapping to study brain plasticity in paretic patients with brain tumours. F Neurol Neurosurg Psychiatry 2000;69:453-63. 